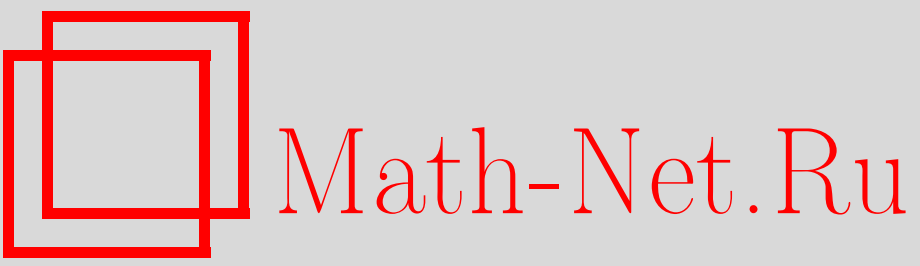

А. Э. Филиппов, Аттракторные свойства физических ветвей решения уравнения ренормализационной группы, ТМФ, 1998, том 117, номер 3, 397-410

DOI: https://doi.org/10.4213/tmf941

Использование Общероссийского математического портала Math-Net.Ru подразумевает, что вы прочитали и согласны с пользовательским соглашением

http://www.mathnet.ru/rus/agreement

Параметры загрузки:

IP: 54.92 .164 .108

26 апреля 2023 г., 02:45:41 
(C) 1998 г.

\author{
А.Э. Филиппов*
}

\title{
АТТРАКТОРНЫЕ СВОЙСТВА ФИЗИЧЕСКИХ ВЕТВЕЙ РЕШЕНИЯ УРАВНЕНИЯ РЕНОРМАЛИЗАЦИОННОЙ ГРУППЫ
}

Исследована глобальная структура фазового портрета локальной версии точного уравнения ренормализационной группы (РГ) для скалярного флуктуирующего поля параметра порядка. Обнаружено, что все физические ветви решения уравнения РГ для неподвижных точек принадлежат аттракторному подпространству, $\mathrm{k}$ которому локальная плотность функционала Гинзбурга-Ландау-Вильсона притягивается при достаточно произвольных затравочных конфигурациях. Установлено, что решение уравнения РГ, соответствующее нетривиальной неподвижной точке, определяющей критическое поведение при фазовом переходе второго рода, является седловой неподвижной точкой данного аттракторного подпространства, разделяющей области притяжения к двум устойчивым решениям, соответствующим высокои низкотемпературному термодинамическим режимам.

\section{ВВЕДЕНИЕ}

Использование уравнения ренормализационной группы (РГ) в теории критических явлений основывается на том, что в критической точке фазового перехода второго рода корреляционный радиус для взаимодействия флуктуаций параметра порядка неограниченно возрастает. В достаточно малой окрестности точки перехода этот радиус превышает любой из микроскопических масштабов длины системы, что позволяет использовать масштабные преобразования для вычисления термодинамических функций.

Предложенная впервые Кадановым [1] идея масштабных преобразований была развита Вильсоном [2] в форме заимствованного из теории поля метода РГ, приспособленного к теории фазовых переходов. В наиболее обшем виде уравнение РГ для этой теории было записано в виде формально точного уравнения в вариационных производных [2, 3] (см. также монографиии [4-7], где метод РГ изложен применительно к различным аспектам теории фазовых переходов и критических явлений).

* Донецкий физико-технический институт Национальной академии наук Украины, Донецк, Украина 
Для решения нелинейного нелокального уравнения РГ было предложено несколько вариантов теории возмушений, наиболее известным из которых является метод $\varepsilon$-разложения [8]. Суть его состоит в построении ряда теории возмущений по степеням разности $\varepsilon=4-d$, где $d$ - размерность пространства. С помощью $\varepsilon$-разложения был достигнут сушественный прогресс в развитии теории критических явлений [2-7]. При этом теория возмушений была продвинута до учета вклада от весьма высоких порядков (см., например, [9]). Однако параметр $\varepsilon$ в действительности не мал и для физически осмысленных задач, для которых $2 \leqslant d \leqslant 3$, лежит в интервале $1 \leqslant \varepsilon \leqslant 2$. Это стимулировало поиски альтернативных вариантов теории, свободных от использования параметра $\varepsilon$. Формально точное (нелокальное) уравнение РГ в вариационных производных позволяло в принципе это сделать. Было показано, что в пределе $\varepsilon \rightarrow 0$ это уравнение имеет ветвь решения, совпадаюшую с аналогичным решением, полученным при непосредственном использовании $\varepsilon$-разложения в теоретико-полевом случае РГ [10, 11]. Вместе с тем точное уравнение РГ может быть существенно упрощено с помощью редукции его к локальной версии [12-16]. Применение $\varepsilon$-разложения к локальному приближению дает результаты, совпадающие с традиционным подходом лишь в первом порядке по $\varepsilon[12]$.

Ниже мы будем ограничиваться простейшим (скалярным и локальным) случаем уравнения РГ (см. ниже уравнение (7)). Можно проверить, что локальное уравнение отвечает низшему порядку разложения по степеням индекса Фишера $\eta$ и соответствует пределу $\eta=0$ [17]. Кроме того, можно построить процедуру решения формально точного исходного уравнения РГ таким образом, что следующие приближения совпадают с соответствующими порядками стандартной теории [7]. Независимо от этого численно найденное решение локального уравнения может быть использовано и в качестве нулевого приближения при построении теории возмущений по нелокальным поправкам без использования разложения по вершинам [18].

Такое разложение дает хорошие результаты уже в первом ненулевом приближении по нелокальностям и, как было указано в работе [18], применимо практически во всем физически интересном интервале размерностей $2 \leqslant d \leqslant 4$. Преимушеством подхода является то, что он оперирует с численно найденной локальной плотностью свободной энергии, формально содержащей все степени параметра порядка $\varphi^{2 k}$. Вместе с тем это делает сравнение результатов для вершин $g_{2 k}$ с получаемыми в теоретико-полевом подходе [9, 19-22] возможным лишь вблизи $d=4$, где оба подхода совпадают в $\varepsilon$-разложении. Хорошее согласие, однако, имеется для физически наблюдаемых критических индексов. В частности, в работе [18] при $n=1$ для размерностей $d=2$, $2.3,2.65,2.95,3,3.23,3.49,3.75,4$ были получены следующие значения индексов $\eta \approx 0.280,0.158,0.075,0.034,0.031,0.134,0.041,0.0,0.0$ и $v \approx 1.093,0.858,0.726$, $0.645,0.640,0.593,0.548,0.519,0.5$. Подробное сравнение этих результатов с критическими индексами, вычисленными в рамках теоретико-полевого подхода, приведено в работе [23].

Кроме того, использование простейшего скалярного приближения соответствует цели данной статьи, посвященной исследованию (глобально) аттракторной природы физических ветвей решения уравнения РГ. Во избежание недоразумений, однако, 
следует заметить, что сами эти решения могут быть получены в гораздо более общем случае. Ранее это было сделано как для многокомпонентного $O(n)$-симметричного поля [24], так и для анизотропных систем [25].

Вычисление критических асимптотик в традищионных схемах РГ выполнено с очень высокой точностью $[9,19-22]$. Поэтому использование иного метода может быть оправдано лишь постановкой качественно новых вопросов, принципиально связанных с непертурбативной природой альтернативного подхода. $\mathrm{K}$ их числу принадлежит вопрос о (глобально) аттракторной природе физических ветвей решения уравнения РГ.

Принято считать, что (седловая) неподвижная точка РГ достигается для произвольной физической системы, претерпевающей фазовый переход второго рода, и это имеет место при очень слабых ограничениях на форму затравочного функционала свободной энергии. Это предположение является одной из гипотез метода РГ и до сих пор практически не исследовано. Следует отметить, что уравнение РГ есть, в свою очередь, частный случай масштабного уравнения для корреляционной функции (в данном случае статистической суммы, рассматриваемой в этом контексте как коррелящионная функция нулевого порядка). Оно входит в качестве уравнения наименьшего порядка в иерархию масштабных уравнений для корреляционных функций произвольного порядка $[26,27]$. Как было показано в работе [26], притяжение его решений к физическим ветвям является ключевым при решении всей системы таких уравнений, описываюших термодинамическое поведение реально наблюдаемых величин.

\section{1. ТОЧНОЕ (ЛОКАЛЬНОЕ) УРАВНЕНИЕ РГ}

Точное уравнение РГ записывается для функционала $H_{I}[\varphi]$, которьй за вычетом квадратичной формы

$$
H_{0}[\varphi]=\frac{1}{\left[2(2 \pi)^{d}\right]} \int d^{d} q G_{0}^{-1}(\mathbf{q})|\varphi(\mathbf{q})|^{2}
$$

совпадает с полным функционалом Гинзбурга-Ландау-Вильсона (ГЛВ) системы $H[\varphi]$,

$$
H_{I}[\varphi]=H[\varphi]-\frac{1}{\left[2(2 \pi)^{d}\right]} \int d^{d} q G_{0}^{-1}(\mathbf{q})|\varphi(\mathbf{q})|^{2},
$$

содержащим все степени флуктуирующего поля $\varphi(\mathbf{q})$. Здесь затравочный пропагатор $G_{0}(\mathbf{q})$ включает фактор $S(\mathbf{q})$,

$$
\frac{1}{2} \int d^{d} q G_{0}^{-1}(\mathbf{q})|\varphi(\mathbf{q})|^{2} \equiv \frac{1}{2} \int d^{d} q \mathbf{q}^{2} S(\mathbf{q})|\varphi(\mathbf{q})|^{2},
$$

определенный таким образом, чтобы обеспечить гладкое обрезание интегралов по импульсам при $|\mathbf{q}| \rightarrow \infty[2,3,7]$. Уравнение РГ в вариационных производных записывается следующим образом:

$$
\begin{aligned}
\frac{\partial H_{I}[\varphi(\mathbf{q})]}{\partial l}= & \frac{V}{2} \int_{\mathbf{q}} \eta(\mathbf{q})+\int_{\mathbf{q}}\left[d+2-\frac{\eta(\mathbf{q}) \varphi(\mathbf{q})}{2}+\mathbf{q} \varphi(\mathbf{q})\left(\nabla_{q} \varphi(\mathbf{q})\right)\right] \frac{\delta H_{I}[\varphi(\mathbf{q})]}{\delta \varphi(\mathbf{q})}- \\
& -\frac{1}{2} \int_{\mathbf{q}} G_{0}^{-1}(\mathbf{q}) \eta(\mathbf{q})|\varphi(\mathbf{q})|^{2}-\frac{d V \partial H_{I}}{\partial V}+ \\
& +\int_{\mathbf{q}} \frac{d S(\mathbf{q})}{d \mathbf{q}^{2}}\left[\frac{\delta^{2} H_{I}[\varphi(\mathbf{q})]}{\delta \varphi(\mathbf{q}) \delta \varphi(-\mathbf{q})}-\frac{\delta H_{I}[\varphi(\mathbf{q})]}{\delta \varphi(\mathbf{q})} \frac{\delta H_{I}[\varphi(\mathbf{q})]}{\delta \varphi(-\mathbf{q})}\right] \equiv R\left[H_{I}\right] .
\end{aligned}
$$


Здесь $l$ - формальное “время” РГ-преобразования, $\eta(\mathbf{q})$ - функция аномальной размерности, совпадаюшая с индексом Фишера при $\mathbf{q}=0$, а интегралы по импульсам для компактности записи определены соотношением

$$
\int_{\mathbf{q}}=(2 \pi)^{-d} \int d^{d} q
$$

Хотя затравочная форма локальной плотности свободной энергии может быть записана в традиционной для непрерывных переходов редуцированной форме

$$
f(\varphi(r) ; l=0)=\frac{\tau \varphi(r)^{2}}{2}+\frac{\beta \varphi(r)^{4}}{4}
$$

реально из-за принципиально существенной перенормировки ее фолуктуациями эффективная свободная энергия в критической области является решением уравнения РГ (3) в неподвижной точке $R\left[H_{I}\right]$ и не может быть редуцирована к конечному числу членов разложения (4).

Кроме того, локальная часть энергии $f(\varphi(r) ; l \neq 0)=\tau(l \neq 0) \varphi(r)^{2} / 2+\beta(l \neq 0) \times$ $\varphi(r)^{4} / 4+\cdots$ содержит вклады от генерируемых в критической области нелокальностей. В низшем приближении можно пренебречь генерацией нелокальных вкладов в ренормированный функционал $H_{I}[\varphi(q)]$, редуцируя его к виду

$$
H[\varphi]=\frac{1}{2} \int_{q} G_{0}^{-1}(\mathbf{q})|\varphi(\mathbf{q})|^{2}+\int d^{d} r f(\varphi(r))=H_{0}[\varphi]+H_{I}[\varphi]
$$

на каждом шаге РГ-процедуры [12-17]. Локальная плотность свободной энергии $f(\varphi(r))$ в этом случае удовлетворяет редуцированному (так называемому "локальному") уравнению РГ

$$
\frac{\partial f(\varphi ; l)}{\partial l}=R[f(\varphi ; l)]
$$

где оператор $R[f(\varphi ; l)]$ для скалярного поля $\varphi$ имеет вид

$$
R[f(\varphi ; l)]=d f(\varphi)-\frac{(d-2)}{2} \varphi \frac{\partial f(\varphi)}{\partial \varphi}+\frac{\partial^{2} f(\varphi)}{\partial \varphi^{2}}-\left[\frac{\partial f(\varphi)}{\partial \varphi}\right]^{2} .
$$

\section{2. АТТРАКТОРНЫЕ СВОЙСТВА ФИЗИЧЕСКИХ ВЕТВЕЙ РЕШЕНИЯ УРАВНЕНИЯ РГ И ГЛОБАЛЬНАЯ СТРУКТУРА ФАЗОВОГО ПОРТРЕТА}

Физические ветви решения (т.е. такие решения, которые сушествуют во всей области изменения переменной $\varphi$ и нарастают при $\varphi \rightarrow \infty)$ для неподвижных точек с $R\left[f^{*}(\varphi ; l \rightarrow \infty)\right]=0$ нелинейного уравнения (6) были получены численно и исследованы в различных аспектах в работах [16-20, 24, 25, 28]. Непосредственной подстановкой в уравнение $(7)$ можно убедиться, что такие решения при $R\left[f^{*}(\varphi ; l \rightarrow \infty)\right]=0$ имеют асимптотику

$$
f^{*}(\varphi ; l \rightarrow \infty)=\frac{\varphi^{2}}{2}+\text { const }
$$


при $\varphi \rightarrow \infty$. Можно проверить также, что такая же асимптотика сохраняется при любом $l$, если затравочная функция $f(\varphi ; 0)$ имела соответствуюшую асимптотику.

Этот факт можно использовать для получения численных решений в произвольньй момент "времени" $l$, притягиваюшихся к неподвижным физическим решениям $f^{*}(\varphi ; l \rightarrow \infty)=\varphi^{2} / 2+$ const. Для этого начальное распределение локальной плотности $f(\varphi ; 0)$ следует брать в форме аппроксимантов Паде:

$$
f_{\mathrm{eff}}(\varphi) \approx \frac{a_{1}+a_{3} \varphi^{2}+a_{5} \varphi^{4}+\cdots}{a_{2}+a_{4} \varphi^{2}+a_{6} \varphi^{4}+\cdots}
$$

даюших возможность задать правильную асимптотику уже в низшем нетривиальном приближении $a_{1}=a_{3}=a_{2 k}>4=0, a_{5}=a_{2}=a_{4} \neq 0$. При этом, однако, затравочные вершины разложения

$$
f(\varphi ; 0)=\sum_{k} g_{2 k}(l=0) \frac{\varphi^{2 k}}{2^{2 k}}
$$

будут несколько отличаться от величин, получаемых в исходном полиномиальном выражении. Проблема граничных условий становится очень существенной, если приходится иметь дело с достаточно произвольным функционалом ГЛВ, который выведен из микроскопической теории и, как правило, не имеет асимптотики $f(\varphi ; l=0)=$ $\varphi^{2} / 2+$ const, совпадаюшей с получаемой в пределе для неподвижной точки $l \rightarrow \infty$, $f^{*}(\varphi ; l \rightarrow \infty)=\varphi^{2} / 2+$ const.

Физические требования дают гораздо более слабые ограничения на функционал свободной энергии, который должен быть определен во всей области изменения поля параметра порядка $-\infty<\varphi<\infty$ и стремиться к бесконечности при $\varphi \rightarrow \infty$. С учетом этого далее удобно переписать локальное уравнение РГ в терминах новой функции $u=\exp (-f)$. Непосредственная подстановка дает

$$
\frac{\partial u(\varphi ; l)}{\partial l}=R[u(\varphi ; l)]=d u(\varphi) \ln [u(\varphi)]-\frac{d-2}{2} \varphi \frac{\partial u(\varphi)}{\partial \varphi}+\frac{\partial^{2} u}{\partial \varphi^{2}}
$$

Граничное условие $f \rightarrow \infty$ при $\varphi \rightarrow \infty$ для функшии $u$ переходит в условие $u \rightarrow 0$ при $\varphi \rightarrow \infty$, которое существенно проще удовлетворить при численных расчетах на конечном массиве $\varphi$. Это позволяет исследовать аттракторные свойства уравнения РГ (11) при достаточно произвольных затравочных формах локальной плотности $f(\varphi ; l=0)$ функционала ГЛВ.

\section{3. МЕХАНИЧЕСКАЯ АНАЛОГИЯ И ФИЗИЧЕСКИЕ ВЕТВИ}

Остановимся прежде всего на статических свойствах уравнения (11). При $\partial u(\varphi ; l) / \partial l=0$ оно может быть переписано в форме уравнения Ньютона

$$
\frac{\partial^{2} u}{\partial \varphi^{2}}=-d u(\varphi) \ln [u(\varphi)]+\frac{d-2}{2} \varphi \frac{\partial u(\varphi)}{\partial \varphi}=-\frac{d V(u)}{d u}+F(u, \varphi)
$$

с потенциалом

$$
V(u)=\frac{1}{2} d u^{2}\left[\ln (u)-\frac{1}{2}\right]
$$


и зависяшей от “времени” $\varphi$ ускоряюшей “силой”

$$
F(u, \varphi)=\frac{d-2}{2} \varphi \frac{\partial u(\varphi)}{\partial \varphi}
$$

Как уже отмечалось в предыдуших публикациях $[17,18,24,25,28]$, статическое уравнение РГ $R[f(\varphi ; l)]=0$ при различных размерностях имеет дискретный набор физических ветвей решения для неподвижных точек (соответствующих различному мультикритическому поведению $[18,24,25])$. То же должно иметь место и для уравнения (12). Механическая аналогия делает этот результат практически очевидным. Действительно, эффективный потенциал (13) имеет минимум при $u=1$, обрашается в нуль в точках $u=0$ и $u=e^{1 / 2}$ и уходит на бесконечность в области $e^{1 / 2}<u<\infty$. Ускоряющая “сила" (14) приводит к уходу фазовых траекторий из долины потенциала $V(u)$. Отражаясь от стенки в области $e^{1 / 2}<u<\infty$, траектории достигают границы устойчивости $u=0$, где функция $\ln u$ выходит с вешественной оси в комплексную плоскость.

Траектории, подходящие к границе в особой точке $u=\partial u(\varphi) / \partial \varphi=0$, отличаются от остальных тем, что достигают ее за бесконечное "время" $\varphi \rightarrow \infty$, поскольку производная от “потенциала" $d V(u) / d u$ и, следовательно (см. уравнение (12)), функция $\partial^{2} u / \partial \varphi^{2}$ в этой точке обрашаются в нуль одновременно с $u=\partial u(\varphi) / \partial \varphi=0$. При фиксированной размерности пространства $d$ и конечной глубине потенциала $V(u)$, а также при заданной зависимости (14) траектория может попасть в точку $u=\partial u(\varphi) / \partial \varphi=0$, совершив ограниченное число отражений от стенок $V(u)$. Набор начальных значений $u_{0}$ задается условием энергетического баланса

$$
\frac{d-2}{2} \int_{u_{0}}^{0} d u \varphi \frac{\partial u(\varphi)}{\partial \varphi}=-V\left(u_{0}\right)
$$

которое после подстановки соответствуюших величин принимает вид

$$
d u_{0}^{2}\left[1-2 \ln u_{0}\right]=(d-2) \int^{\infty} d\left(\varphi^{2}\right)\left[\frac{\partial u\left(\varphi_{0}\right)}{\partial \varphi}\right]^{2},
$$

где функция $\partial u\left(\varphi_{0}\right) / \partial \varphi$ является решением уравнения (12) при начальном $u$, равном данному $u_{0}$. Интеграл в правой части соотношения (16) положителен и при $d>2$ имеет по крайней мере два пересечения с положительной частью функции $d u_{0}^{2}\left[1-2 \ln u_{0}\right]$ (при $u_{0}>1$ и $u_{0}<1$, соответственно). Непосредственной подстановкой можно убедиться, что первое из этих решений совпадает с так назьваемой “высокотемпературной неподвижной точкой” $u=\exp \left[\varphi^{2} / 2+1 / d\right]$ (и соответственно $\left.f=\varphi^{2} / 2+1 / d\right)[3,4]$. Формально соотношение (16) дает также решение и при $u_{0}<1$, которое определяет физическую ветвь для критической неподвижной точки. Однако ее вычисление требует численного решения. В этом случае уже выполненное ранее прямое численное решение исходного уравнения [18] является более предпочтительным. Тем не менее аналогия с механическим движением частицы в эффективном потенциале дает ключ к пониманию глобальных аттракторных свойств зависящего от времени уравнения РГ, которые будут исследованы ниже. 


\section{4. РГ-ПРЕОБРАЗОВАНИЕ КАК РЕЛАКСАЦИЯ В НЕОДНОРОДНОМ ПРОСТРАНСТВЕ}

Вернемся к зависящему от времени уравнению РГ в терминах функции $u$. Из уравнения непосредственно видно, что оно также содержит потенциал $V(u)$ (однако с отрицательным знаком $\left.V_{-}(u)=-d u^{2}[\ln (u)-1 / 2] / 2\right)$. В отсутствие связанного с изменением масштаба слагаемого $(d-2) \varphi[\partial u(\varphi) / \partial \varphi] / 2$ это уравнение генерируется следующим производящим функционалом:

$$
\begin{aligned}
H[u(\varphi)] & =\frac{1}{2} \int d \varphi\left\{\left[\frac{\partial u(\varphi)}{\partial \varphi}\right]^{2}+V_{-}(u)\right\}= \\
& =\frac{1}{2} \int d \varphi\left\{\left[\frac{\partial u(\varphi)}{\partial \varphi}\right]^{2}+d u^{2}\left[\ln (u)-\frac{1}{2}\right]\right\}=\int d \varphi E\left\{\frac{\partial u}{\partial \varphi} ; u\right\}
\end{aligned}
$$

и имеет форму релаксационного уравнения

$$
\frac{\partial u(\varphi ; l)}{\partial l}=-\frac{\delta H[u(\varphi)]}{\delta \varphi}
$$

которое совпадает с уравнением Ландау-Халатникова, используемым в кинетическом подходе к теории фазовых переходов [5]. В данном случае уравнение (18) описывает притяжение к системе аттракторных решений, задаваемых уравнением

$$
\frac{\partial u^{*}(\varphi ; l \rightarrow \infty)}{\partial l}=-\frac{\delta H\left[u^{*}(\varphi)\right]}{\delta \varphi}=0,
$$

которое, как мы видели, совпадает с уравнением Ньютона и имеет, следовательно, однопараметрическое семейство интегралов

$$
E\left\{\frac{\partial u^{*}}{\partial \varphi} ; u^{*}\right\}=\frac{1}{2}\left\{\left[\frac{\partial u^{*}(\varphi)}{\partial \varphi}\right]^{2}+d u^{*_{2}}\left[\ln \left(u^{*}\right)-\frac{1}{2}\right]\right\}=\text { const }
$$

задаваемых уровнями энергии эффективного потенщиала.

Можно проверить, что условию $u^{*} \rightarrow 0$ при $\varphi \rightarrow \infty$ в данном случае удовлетворяет единственный интеграл, получаемый из условия (20) при $E\left\{\partial u^{*} / \partial \varphi ; u^{*}\right\}=$ const $=0$ и совпадаюший с уже упомянутой выше "высокотемпературной неподвижной точкой" $u^{*}=\exp \left[\varphi^{2} / 2+1 / d\right]$, что, в свою очередь, согласуется с известным фактом единственности такой точки в случае равенства нулю слагаемого $(d-2) \varphi[\partial u(\varphi) / \partial \varphi] / 2$ для двумерных систем $d=2[16-18,24,25,28]$.

При $d \neq 2$ производящий функционал меняется:

$$
H[u(\varphi)]=\int d \varphi \exp \left[-\frac{(d-2) \varphi^{2}}{4}\right]\left\{\frac{1}{2}\left[\frac{\partial u(\varphi)}{\partial \varphi}\right]^{2}+V_{-}(u)\right\} .
$$

В присутствии множителя $\exp \left[-(d-2) \varphi^{2} / 4\right]$ в функццинале $H[u(\varphi)]$ пространство параметра порядка $\varphi$ становится неоднородным и система уже не имеет интегралов (20). Порождаемое этим множителем слагаемое $(d-2) \varphi[\partial u(\varphi) / \partial \varphi] / 2$ зависит 
от переменной $\varphi$ явно и вносит наиболее сильный вклад в эволюцию $\partial u(\varphi ; l) / \partial l$ в окрестностях максимумов произведения $\varphi \partial u(\varphi) / \partial \varphi$, в которых эта комбинация знакоопределена $(d-2) \varphi[\partial u(\varphi) / \partial \varphi] / 2>0$. Ее вклад в $\partial u(\varphi ; l) / \partial l$, в свою очередь, отрицателен и может фиксировать локальньй минимум функции $u(\varphi ; l)$, если затравочная функция $\partial u(\varphi ; l=0)$ уже имела такой минимум. В конечном счете именно этот эффект и приводит к появлению решения, не совпадаюшего ни с $u(\varphi ; l \rightarrow \infty)=0$, ни с $u(\varphi ; l \rightarrow \infty)=\exp \left[-\varphi^{2} / 2+1 / d\right]$.

\section{5. ОБСУЖДЕНИЕ РЕЗУЛЬТАТОВ}

Получить аналитическое решение уравнения (11) сегодня не представляется возможным. Результаты его численного исследования суммированы на рис. 1-7 и обсуждаются ниже.

Функционал ГЛВ может иметь произвольную форму, удовлетворяюшую весьма слабому ограничению $u(\varphi ; l=0) \rightarrow 0$ при $\varphi \rightarrow \infty$. Для того чтобы генерировать достаточно произвольные начальные условия, был использован источник случайного шума. При этом для четных затравочных функционалов использовалась симметризация

$$
u(\varphi ; l=0)=\frac{1}{2}[u(\varphi ; l=0)+u(-\varphi ; l=0)],
$$

устраняюшая нечетные слагаемые, порождаемые случайным источником. Проекции линий тока на различные подпространства зависящих от РГ-“времени" $l$ коэффициентов разложения (так называемых “вершин” $g_{2 k}(l)$ )

$$
f(\varphi ; l)=-\ln [u(\varphi ; l)]=\sum_{k} g_{2 k}(l) \frac{\varphi^{2 k}}{2^{2 k}}
$$

вычислялись численно для текуших конфигураций эволюционирующего массива $u(\varphi ; l)$, содержащего $10^{3}$ элементов.

На рис. 1а показаны три характерных типа (А, В и С) эволюции функции $u(\varphi ; l)$, стартуюшей с трех типичных, но приводящих к качественно различному поведению фазовых траекторий, начальных распределений локальной плотности $f(\varphi ; l)=$ $-\ln [u(\varphi ; l)]$. Все три типа сопоставлены с проекциями их фазовых траекторий на подпространство $\left(g_{4}(l), g_{6}(l)\right)$ (рис. 1б-г).

Нетрудно видеть, что на начальном этапе эволюции релаксационный процесс стремительно сглаживает все коротковолновые особенности функции $u(\varphi ; l)$, порожденные конкретными начальными условиями. На фазовом портрете этому процессу соответствует быстрое притяжение фазовых траекторий к окрестности универсальной аттракторной линии, называемой траекторией “большой реки" (жирная линия на рис. 1a) [28].

Интересно отметить, что такое поведение очень близко к тому, которое ожидалось априори в рамках нелинейного диффузионного уравнения, первоначально использованного Вильсоном при построении точного уравнения РГ [2]. Упомянутое различие 

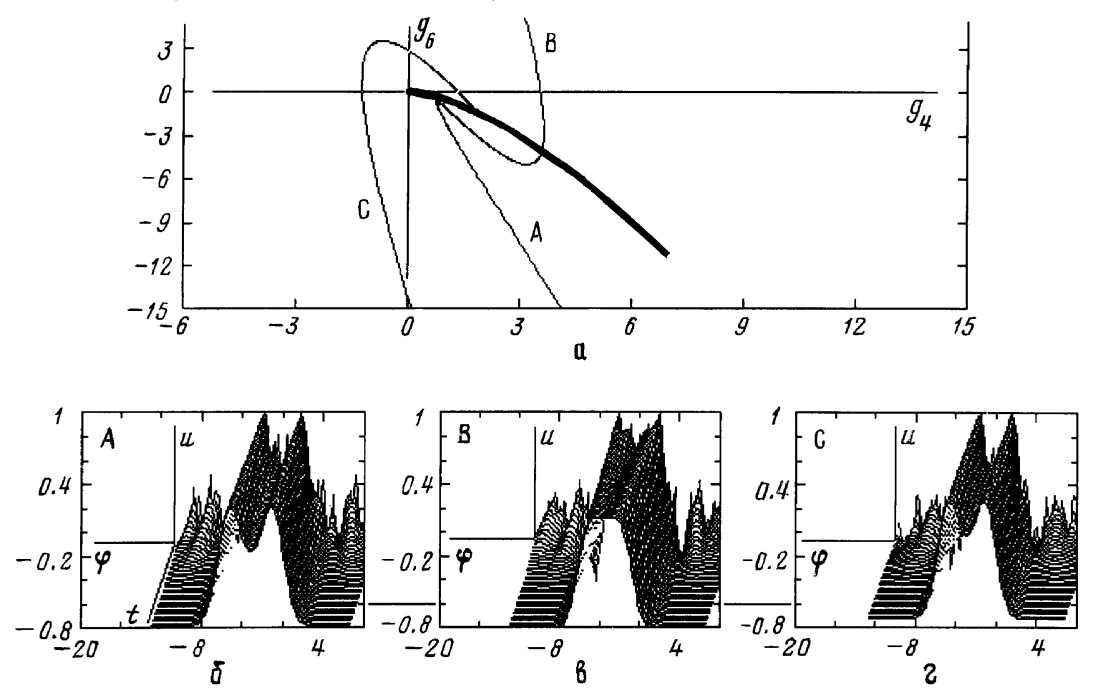

Рис. 1

в темпе эволюции вдали от "большой реки" и вблизи от нее может быть установлено численно посредством расчета интегрального потока, определенного вслед за работами $[16,17]$ посредством скалярного произведения

$$
\left\|\frac{\partial u(\varphi ; l)}{\partial l}\right\| \equiv\left\langle\frac{\partial u(\varphi ; l)}{\partial l} \mid \frac{\partial u(\varphi ; l)}{\partial l}\right\rangle,
$$

задаваемого интегралом от $[\partial u(\varphi) / \partial \varphi]^{2}$ по всей области изменения $\varphi$, вычисленным с весом $w(\varphi)=\exp \left[-(d-2) \varphi^{2} / 4\right]$ :

$$
\left\langle\frac{\partial u(\varphi ; l)}{\partial l} \mid \frac{\partial u(\varphi ; l)}{\partial l}\right\rangle=\int d \varphi \exp \left[-\frac{d-2}{4} \varphi^{2}\right]\left[\frac{\partial u(\varphi)}{\partial \varphi}\right]^{2} .
$$

На притягивающем подпространстве “большой реки” система продолжает медленно эволюционировать к одному из трех неподвижных решений. Приведенные на рис. 1 случаи отражают все три возможности:

1) низкотемпературная область $g_{2}<g_{2}^{*}$ (тип А, рис. 1б);

2) высокотемпературная область $g_{2}>g_{2}^{*}$ (тип В, рис. 1в);

3) критическая точка $g_{2}=g_{2}^{*}$ (тип С, рис. $1 г$ ).

На рис. 2 показано соотношение между решением $u^{*}(\varphi ; l \rightarrow \infty)$ в критической точке (изображенным жирной линией) и линиями уровня потенциала

$$
V(u)=\frac{1}{2}\left\{\left[\frac{\partial u^{*}(\varphi)}{\partial \varphi}\right]^{2}+d u^{* 2}\left[\ln \left(u^{*}\right)-\frac{1}{2}\right]\right\}
$$

совпадаюшими при $d=2$ с интегралами статического уравнения

$$
E\left\{\frac{\partial u^{*}}{\partial \varphi} ; u^{*}\right\}=\frac{1}{2}\left\{\left[\frac{\partial u^{*}(\varphi)}{\partial \varphi}\right]^{2}+d u^{* 2}\left[\ln \left(u^{*}\right)-\frac{1}{2}\right]\right\}=\text { const } .
$$




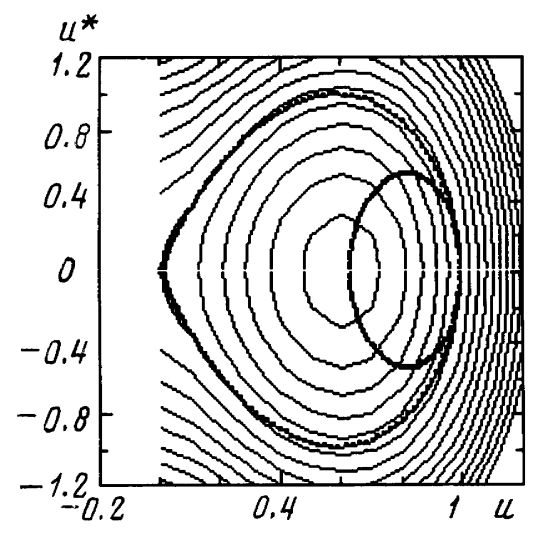

Рис. 2
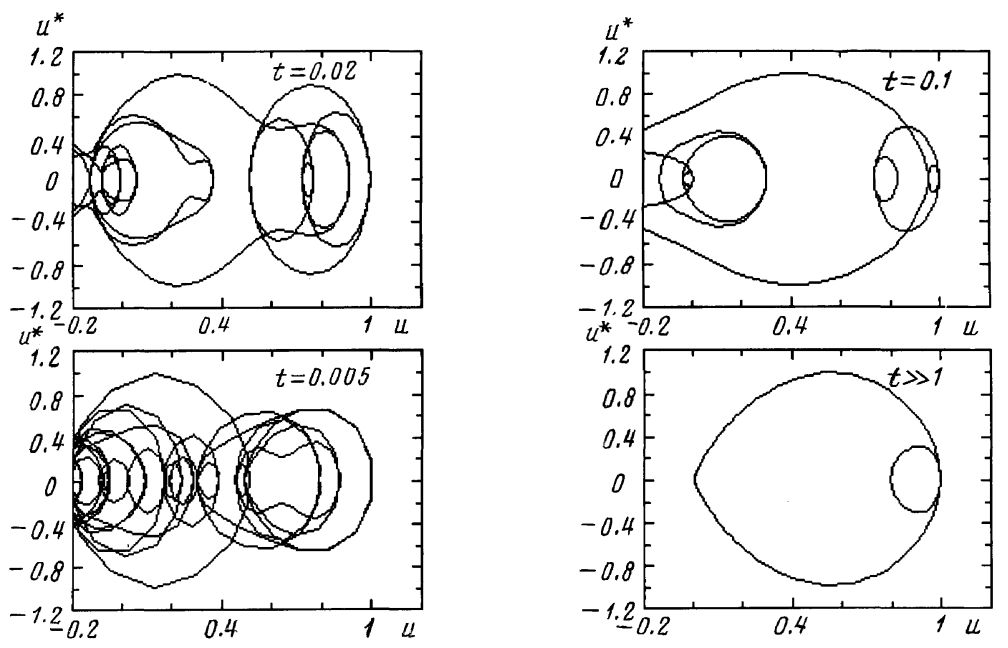

Рис. 3

Большая петля кривой $u^{*}(\varphi ; l \rightarrow \infty)$ на этом рисунке асимптотически приближается к линии $E\left\{\partial u^{*} / \partial \varphi ; u^{*}\right\}=0$ (соответствуюшей, как уже обсуждалось вьше, решению $\left.u(\varphi ; l \rightarrow \infty)=\exp \left[-\varphi^{2} / 2+1 / d\right]\right)$, тогда как малая внутренняя петля отражает присутствие локального минимума функции $u^{*}(\varphi ; l \rightarrow \infty)$, порожденного неоднородным слагаемым $(d-2) \varphi[\partial u(\varphi) / \partial \varphi] / 2$ в уравнении РГ.

Рис. 3 иллюстрирует глобальную устойчивость и быструю сходимость решений к описанной вьше конфигурации рис. 2. Для наглядности последовательные моменты времени, для которых представлены текушие конфигурации $u(\varphi ; l)$, выбраны с экспоненциально нарастаюшим интервалом между ними.

Будучи седловой, критическая неподвижная точка разделяет аттракторное подпространство уравнения РГ на две области притяжения к глобально устойчивым высоко- 


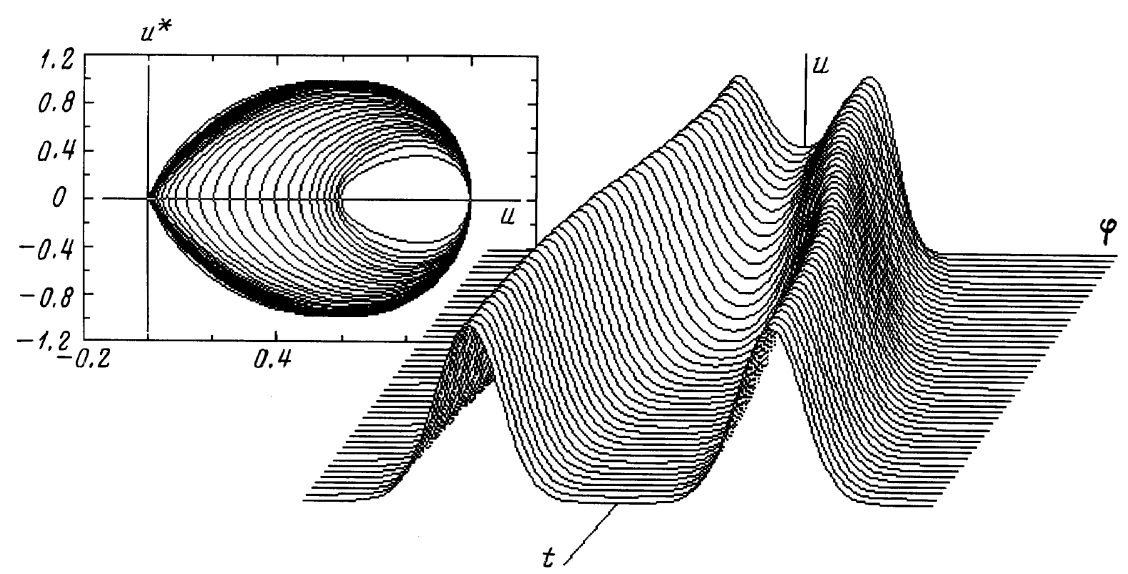

Рис. 4

и низкотемпературному режимам. Этот результат кажется настолько очевидным, что фактически закладывался как одна из основных гипотез применимости уравнения РГ к исследованию критического поведения при фазовых переходах [2-7]. Однако он никогда ранее не был установлен для уравнения РГ, содержашего полньй набор вершин $g_{2 k}(l)$.

Еще в меньшей степени был прояснен вопрос о том, что происходит при уходе фазовых траекторий в низкотемпературную область. Мы обнаружили, что система здесь притягивается к новому решению, которое асимптотически совпадает с парой “высокотемпературных" решений. Процесс такого распада показан на рис. 4 в форме развертки по времени.

На фазовом портрете (показанном также в изометрии на рис. 5) процессу разбегания пары таких решений соответствует деформация внутренней петли $u(\varphi ; l)$, приближаюшейся к внешней асимптотике $u=\exp \left[-\varphi^{2} / 2+1 / d\right]$ при $l \rightarrow \infty$. В пределе $l \rightarrow \infty$ оба устойчивых режима отличаются лишь количеством петель (одна или две для высоко- и низкотемпературного режимов, соответственно).

В низкотемпературном случае мы имеем дело с парой движушихся нелинейных (солитоноподобных) возбуждений, которые могут быть аппроксимированы следуюшим выражением:

$$
\begin{aligned}
u(\varphi ; l \rightarrow \infty) & =a(l)\left\{\exp \left\{-\frac{[\varphi-b(l)]^{2}}{2}+\frac{1}{d}\right\}+\exp \left\{-\frac{[\varphi+b(l)]^{2}}{2}+\frac{1}{d}\right\}\right\}= \\
& =u_{-}(\varphi ; l)+u_{+}(\varphi ; l) .
\end{aligned}
$$

Численное решение показывает, что скорость обоих крыльев $u_{-}(\varphi ; l)$ и $u_{+}(\varphi ; l)$ обрашается в нуль при $l \rightarrow \infty$. К сожалению, найти точное неподвижное решение для них не удается. Однако представление его парой движущихся волн (26) дает хорошую аппроксимацию для этого решения. Учитьвая, что в окрестности максимума $u_{+}(\varphi ; l)$ 

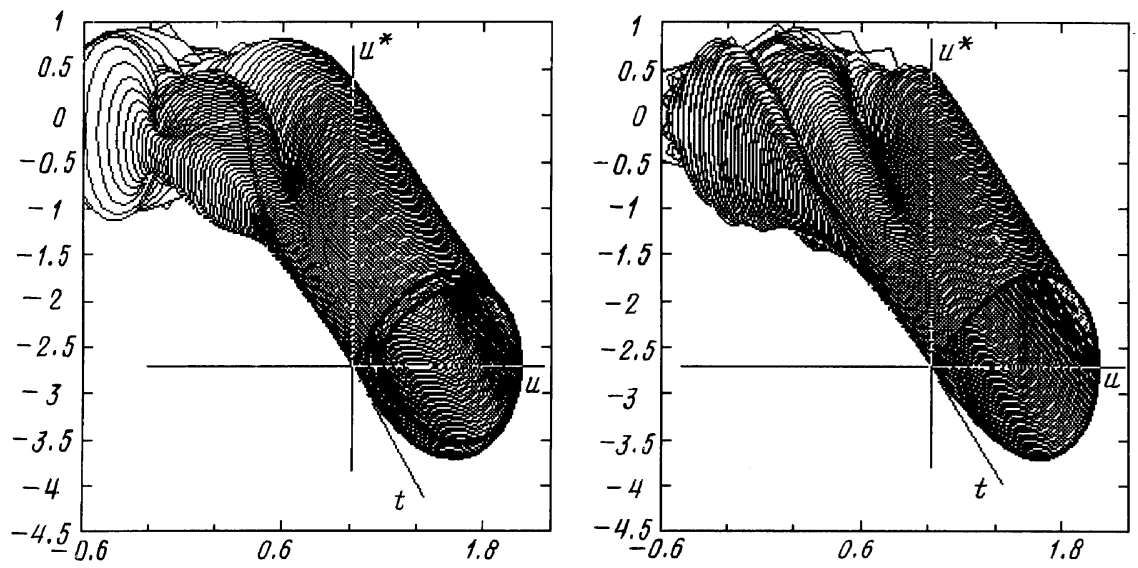

Рис. 5
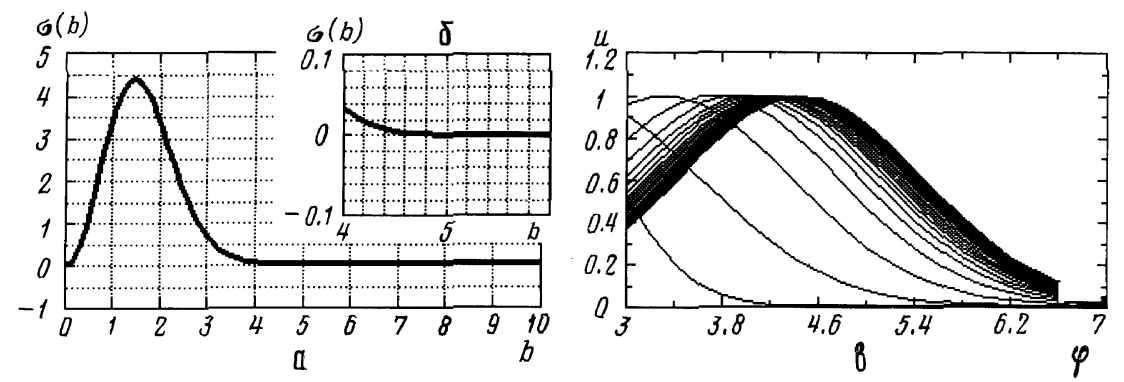

Рис. 6

величина $u_{-}(\varphi ; l)$ мала, в этой области можно записать приближенно

$$
\frac{\partial u(\varphi ; l)}{\partial l}=d\left[u_{-} \ln \left(\frac{u_{+}}{u_{-}}\right)+u_{+} \ln \left(1+\frac{u_{-}}{u_{+}}\right)\right] \approx d u_{-} \ln \left(\frac{u_{+}}{u_{-}}\right)
$$

Подставляя теперь $u_{+}$и $u_{-}$в это соотношение и выполняя простые преобразования, имеем уравнение, определяющее скорость эволюции коэффициента $a(l)$ со временем через текушее положение максимумов $b(l)$ :

$$
\frac{\partial a(l)}{\partial l} \approx 2[b(l)]^{2} \exp \left\{-\frac{[b(l)]^{2}}{2}\right\}
$$

Функция $\sigma(b)=2 b^{2} \exp \left\{-b^{2} / 2\right\}$, показанная на рис. 6а и 6б, быстро стремится к нулю (и становится сопоставимой с численной точностью) в области $4<b=b_{c}<5$. Это дает искомую оценку для интервала остановки пары разбегающихся нелинейных возбуждений $u_{-}(\varphi ; l)$ и $u_{+}(\varphi ; l)$, которая, как видно из рис. 6в, очень хорошо согласуется с результатом численного счета. 


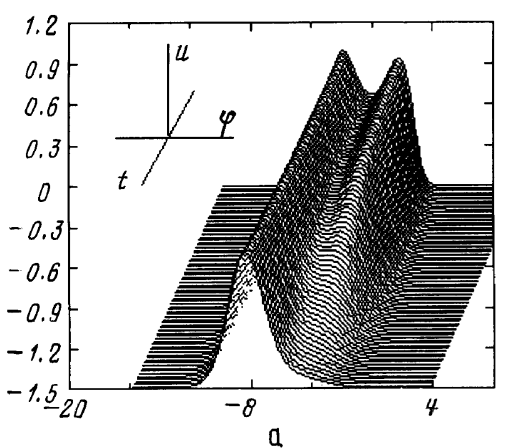

a

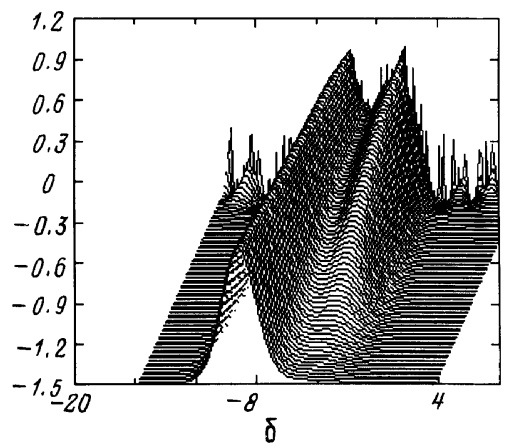

Рис. 7

Для физической плотности свободной энергии двум максимумам $u_{-}(\varphi ; l)$ и $u_{+}(\varphi ; l)$ функции $u(\varphi ; l)=\exp [-f(\varphi ; l)]$ соответствует пара минимумов, определяюших равновесные значения параметра порядка при температуре ниже критической. Этот результат также кажется совершенно естественным с точки зрения "наивной" теории критических явлений, однако также никогда ранее не был подтвержден для уравнения РГ.

Столь же естествен вопрос об асимптотическом поведении решений при снятии симметрийного ограничения $f(\varphi ; l)=f(-\varphi ; l)$, которое происходит, в частности, при наложении внешнего поля [2-7]. Еше более существенным является вопрос о поведении решений в общем случае отсутствия симметрии $f(\varphi ; l)=f(-\varphi ; l)$ в функционале ГЛВ при произвольной структуре затравки $f(\varphi ; l=0)$. В данном подходе, когда глобальная устойчивость решений для функции $u(\varphi ; l)=\exp [-f(\varphi ; l)]$ автоматически обеспечивается граничным условием $u(\varphi \rightarrow \infty ; l) \rightarrow 0$, для ответа на этот вопрос достаточно опустить симметризацию случайного начального распределения $u(\varphi ; l=$ $0)=[u(\varphi ; l=0)+u(-\varphi ; l=0)] / 2$.

Типичная эволющия распределения $u(\varphi ; l)$ с нарушенной симметрией показана на рис. 7 а и $7 б$ при отсутствии и наличии случайного шума в затравке $u(\varphi ; l=0)$, соответственно. Как и следовало ожидать, при малых отклонениях от симметричного случая эти решения показывают хорошо известную неустойчивость критической точки, обусловленную положительностью первого нечетного собственного числа $[2,3]$. Последуюшее поведение сводится к притяжению решения к одной из описанных выше гауссовых ветвей со смещенным центром $a(l) \exp \left\{-[\varphi \pm b(l)]^{2} / 2+1 / d\right\}$, что вполне ожидаемо для системы, релаксируюшей к аттрактору в (неоднородном) пространстве $\varphi$. Вместе с тем этот результат не может быть получен в рамках РГ, использующей теорию возмущений по степеням нечетной добавки к функционалу ГЛВ.

Автор благодарит Ж.-П. Бадиали (J.-P. Badiali) и В. Руссиера (V. Russier) за то, что они стимулировали интерес к проблеме глобальной устойчивости уравнения РГ, а также за гостеприимство в Университете Пьера и Марии Кюри (Париж-VI) и многочисленные интенсивные дискуссии, благодаря которым работа приобрела свою 
настояшую форму.

Работа частично поддержана Фондом фундаментальных исследований Украины (договор № $\Phi 4 / 72-97$, проект № 2.4/199) и Программой сотрудничества НАН Украины и CNRS France $\left(n^{0}\right.$ de dossier 3060$)$.

\section{Список литературы}

[1] L. P. Kadanoff. Physics. 1966. V. 2. P. 263.

[2] К. Вильсон, Дж. Когут. Ренормализационная группа и $\varepsilon$-разложение. М.: Мир, 1975.

[3] F. J. Wegner. The critical state, general aspects. In: Phase transitions and critical phenomena. New York-London: Academ. Press, 1976. P. 8.

[4] ШІ.-К. Ма. Современная теория критических явлений. М.: Мир, 1980.

[5] А.З. Паташинский, В. Л. Покровский. Флуктуационная теория фазовых переходов. М.: Наука, 1982.

[6] Ю.А. Изюмов, Ю.Н. Скрябин. Статистическая механика магнитоупорядоченных систем. М.: Наука, 1987.

[7] Ю.М. Иванченко, А.А. Лисянский, А. Э. Филиппов. Флуктуационные эффекты в системах с конкурирующими взаимодействиями. Киев: Наукова думка, 1989.

[8] K. G. Wilson. Phys. Rev. B. 1971. V. 4. P. 3174

[9] S. G. Gorishny, S. A. Larin, F. V. Tkachov. Phys. Lett. A. 1976. V. 101. P. 120.

[10] J. Rudnick. Phys. Rev. Lett. 1975. V. 3. P. 438.

[11] P. Shukla, M.S. Green. Phys. Rev. Lett. 1975. V. 3. P. 436.

[12] V. I. Tokar. Phys. Lett. A. 1984. V. 104. P. 135.

[13] A. Hasenfratz, P. Hasenfratz. Nucl. Phys. B. 1986. V. 270. P. 687.

[14] G. Felder. Commun. Math. Phys. 1987. V. 11. P. 101.

[15] G. R. Golner. Phys. Rev. B. 1986. V. 33. P. 7863.

[16] G. Zumbach. Phys. Rev. Lett. 1993. V. 71. P. 2421.

[17] G. Zumbach. Phys. Lett. A. 1994. V. 190. P. 225.

[18] А.Э. Филиппов, А. В. Радиевский. ЖЭТФ. 1992. Т. 102. С. 1899.

[19] N. V. Brilliantov, J.P. Valeau, C. Bagnuls, C. Bervillier. Renormalization group, critical behavior, field theory, and continuum limit: a discussion. Preprint Service de Phys. Theor. C.E. Saclay. Paris: Saclay, 1997.

[20] G. A. Baker, B. G. Nickel, D. I. Meiron. Phys. Rev. B. 1978. V. 17. P. 1365.

[21] A. I. Sokolov, E. V. Orlov, V. A. Ul'kov. Phys. Lett. A. 1997. V. 227. P. 255.

[22] R. Guida, J. Zinn-Justin. Nucl. Phys. B. 1997. V. 489. P. 626.

[23] Ю. Головач. ТМФ. 1993. Т. 96. С. 482.

[24] A. Э. Филиппов. ТМФ. 1992. Т. 91. С. 320.

[25] S. A. Breus, A.E. Filippov. Physica A. 1993. V. 192. P. 486.

[26] А. Э. Филиппов. ЖЭТФ. 1995. Т. 108. С. 1429.

[27] Ю. М. Иванченко, А. А. Лисянский, А. Э. Филиппов. ТМФ. 1990. Т. 84. С. 223.

[28] C. Bagnuls, C. Bervillier. Phys. Rev. Lett. 1988. V. 60. P. 1464.

Поступила в редакцию 18.IX.1997 г., после доработки 17.VII.1998 г. 